\title{
Ethnopharmacological and Precliical Studies on Hepatosaab Used In the Management of Leishmaniasis
}

\author{
Amodu B ${ }^{1}$, Itodo S.E ${ }^{2}$, Musa, D.E ${ }^{3}$ \\ 1 Halamin Herbal centre, 10 George Innih Crescent, Apo District, Abuja Nigeria \\ ${ }^{2}$ Department of Histopathology and Cytology, Jos University Teaching Hospital (JUTH) Jos Nigeria \\ ${ }^{3}$ Halamin Herbal centre, 10 George Innih Crescent, Apo District, Abuja Nigeria
}

\begin{abstract}
Leishmaniasis is a generally neglected disease, particularly in terms of new drug development for which there is little potential for financial return. Although there have been significant advances in treatment for leishmaniasis in the past decade, these developments cannot match the scale of more profitable drugs, more so that the attendant high toxicity and side effects associated with the conventional orthodox supplements left much to be desired. HEPATOSAAB, an ethnomedicinal poly herbal formulation from the stable of Halamin Herbal Centre has been found to cure Visceral Leishmaniasis which works by inducing high levels of certain cytokines to positively affect macrophage populations and up-regulates the expression of MHC class II molecules on the surface of the macrophage. It is clearly demonstrated that this inexpensive, simple, effective and non toxicethnomedicinal poly herbal formulation has a promising curative role in the treatment of Visceral Leishmaniasis. The real challenge now will be proper translation, distribution and expansion of the advances made; it is essential that the new treatment options become truly accessible, not simply available, in endemic areas so that they may promote healing and save lives.
\end{abstract}

Keyword: Hepatosaab, Leishmaniasis, MHC class II, Ethnomedicinal

\section{Introduction}

Leishmaniasis is a disease caused by protozoanparasites that belong to the genus Leishmania and is transmitted by the bite of certain species of sand fly (subfamily Phlebotominae). Although the majority of the literature mentions only one genus transmitting Leishmania to humans (Lutzomyia) in America, a 2003 study by Galati suggested a new classification for American sand flies, elevating several subgenera to the genus level. Elsewhere in the world, the genus Phlebotomus is considered the vector of leishmaniasis.

Visceral leishmaniasis is the most severe form of leishmaniasis. It is caused by L. donovani and L. infantum. In endemic areas, the disease is more chronic with young adults and children being more commonly infected. About twice as many male are infected than females. In epidemics, all age groups are susceptible (except those with acquired immunity), and the disease is often acute. Without treatment, VL is usually fatal. Significant advances have been made in the treatment of visceral leishmaniasis.

Symptoms in chronic VL include irregular fever, splenomegaly, hepatomegally, and, or, lymphadenopathy, loss of weight with wasting, diarrhea, low white cell and platelet counts, and anaemia. Skin changes are common.

In acute VL there is splenomegaly, high undulating fever, chills, profuse sweating, rapid weight loss, fatigue, anaemia, and leucopenia. Often there is epistaxis and bleeding from the gums.

In active visceral leishmaniasis there is poor cell-mediated immune response and therefore the parasites multiply rapidly. There is, however, a humoral response with large amounts of polyclonal non-specific immunoglobulin especially IgG being produced and also specific anti-leishmanial antibody. Patients who have recovered from reinfection but relapses can occur, particularly in those coinfected with HIV

Most forms of the disease are transmissible only from animals (zoonosis), but some can be spread between humans. Human infection is caused by about 21 of 30 species that infect mammalsthe different species are morphologically indistinguishable, but they can be differentiated by isoenzyme analysis, DNA sequence analysis, or monoclonal antibodies.

Although there have been significant advances in treatment for leishmaniasis in the past decade, but the attendant high toxicity and side effects associated with the conventional orthodox supplements left much to be desired. However, the liver and the spleen which are the culprit organs that visceral leishmaniasis affect can be toned by some herbal extracts. One of such polyherbal formulations used for various ethnomedicinal purposes in Nigeria including the treatment of Hepatitis $B$ and $C$ is HEPATOSAAB $^{\mathrm{R}}$. The constituents of HEPATOSAAB $^{\mathrm{R}}$ include; sesamumindicatum, Vernoniaamygdalina, Aloe barbadensis, Saccharumofficinarum, Allium sativum, Amaranthusandcaudatus. The medicinal uses of the constituents of HEPATOSAAB ${ }^{\mathrm{R}}$ are well documented in literature (Dalziel 1937). 
But like most traditional medicines in Africa, little or no scientific information is available on this polyherbal component preparation that is akin to the Chinese traditional medicine (TCM) or the India Ayurvedic preparation.

These active constituents are believed to strengthen the immune system through many cytokines and chemokines regulations. With the abundant presence of tannins, phlobatannins, flavonoids, steroids, terpenoids, saponins and cardiac glycosides, which are the most important bioactive constituents of medicinal plants, the poly herbal preparation is able to regenerate the hepatic and pancreatic cells respectively within a span of twelve weeks.

Leishmaniasis infection can be prevented by Preventingsandfly bites.

This study is therefore designed to determine the clinical activities of HEPATOSAAB ${ }^{\mathrm{R}}-$ Vernoniaamygdalina, Aliumsativum, Ossimungratissum and Aloe barbadensis on visceral leishmaniasis andto create awareness to the public and other healthcare workers on the importance of preventing the bite of sand flies.

\section{Methodology}

STUDY DESIGN: The study is a descriptive cross-sectional survey.

STUDY AREA: This study was carried out at the Medical Laboratory Department of Halamin Chemicals Nigeria Limited, Abuja F.C.T. This is a pharmaceutical company that specializes in the research and manufacture of supplements of African origin indicated for the management and treatment of various non communicable diseases and some communicable diseases. Approval was sought and obtained from the inventor of Halamin herbal products before this work was carried out.

STUDY POPULATION: Study population was made of suspected cases ofVisceralLeishmaniasis.

Clients within the age bracket 0 month to 59 years, that came to seek medicalattention in Halamin Chemicals Nigeria Limited centre, Abuja from March, 2012 to August, 2012. Suspected cases comprised of clients that presented with fever and any of the following: heart burn, malaise, nausea, vomiting, dark colored urine or jaundice.

DATAL COLLECTION: Social-demographic data including age, sex, presenting complaints, relevant signs elicited and orthodox drugs pretreatment were obtained from the clients.

SPECIMEN COLLECTION: After obtaining the relevant information and explaining to the clients the test procedures, $2 \mathrm{ml}$ of blood was collected aseptically through venepuncture using sterile syringe and needle following application of tourniquet. The surface of the skin was disinfected with methylated spirit on cotton wool and allowed to dry. The blood was transferred to an EDTA container and centrifuged for 15 minutes at 1000rpm. The plasma above the buffy coat was carefully removed using a Pasteur pipette. The buffy coat was carefully transferred to a slide and mixed. A thin smear was made, air-dried and fixed with methanol for 2 minutes. The prepared smear was stained by Giemsa technique and dried. The prepared slide was examined microscopically using 40x and 100x objective lenses respectively after dropping an oil immersion.

The test was done according to the District Laboratory Practice in Tropical Countries manual.

\section{Results}

The results of the findings of visceral leishmaniasis and the frequency and clearance level of thevisceralleishmaniasis are presented below:

An assessment of the prevalence level of visceralleishmaniasis clients shows that nearly all age groups are affected. However, it was observed that the highest occurrence of positive cases was recorded within the active age group of 20-29 years; no positive case was recorded in the age group of 0-9 years.

Table 2 shows that the number of negative cases outweighed the positives with $66.5 \%$ and $33.5 \%$ respectively. The males' gender recorded highest positive number with $64.91 \%$, while females recorded only $35.08 \%$.

According to table 3 , it could succinctly be said that there was a $100 \%$ clearance of VL after three weeks use of the dietary supplement

Table 1: VISCERAL LEISHMANIASIS (VL) in different Ages (CROSS TABULATION)AGE GROUP (YEAR)

\begin{tabular}{|c|c|c|c|c|c|c|c|c|}
\hline Occurrence of VL & & $0-9$ & $10-19$ & $20-29$ & 30-39 & $40-49$ & $50-59$ & TOTAL \\
\hline Positive & $\mathbf{0}$ & 9 & 49 & 5 & 3 & 2 & \multicolumn{2}{|c|}{$40 \%(68)$} \\
\hline Negative & & 10 & 11 & 21 & 26 & 27 & 7 & $60 \%(102)$ \\
\hline Total & & 10 & 20 & 70 & 31 & 30 & 9 & $100 \%(170)$ \\
\hline
\end{tabular}


Table 2: LEISHMANIASIS in respect to sex (CROSS TABULATION)

\begin{tabular}{|c|c|c|c|}
\hline Occurrence of VL & Male & $\frac{\text { SEX }}{\text { Female }}$ & Total \\
\hline Positive & 37 & 20 & 57 \\
\hline Negative & 64 & 49 & 113 \\
\hline Total & 101 & 69 & 170 \\
\hline
\end{tabular}

Table 3: CLEARANCE LEVEL OF VL AFTER THREE MONTHS USE OF THE DIETARY SUPPLEMENTS

\begin{tabular}{llllc}
\hline & Frequency & percent & valid percent & cumulative percent \\
VL & 0 & 0 & 0 & 0 \\
Total & 0 & 100 & 100 & \\
\hline
\end{tabular}

\section{Discussion}

Leishmaniases are endemic in 88 countries with an estimated 350 million people at risk of infection. The overall prevalence of the leishmaniases is estimated at 12 million cases with 0.5 million new visceral leishmaniasis cases per year.

Generally from the research carried out in the study area, it can be succinctly said that nearly all age group are affected, this is due to the resilience nature of the parasite surviving in almost all body fluid.

\section{CONCLUSION}

HEPATOSAAB with Vernoniaamygdalina as it active ingredient had been used in traditional medicine for the treatment of various ailments (Igileet al., 2004), it has been used to treat gastrointestinal disorders, haematoma, malaria, inflammation, cancer, ataxia and other neglected tropical diseases. sequel to this finding, it has become clear that leishmaniasis and other neglected tropical diseases can be effectively managed with the concoctions infused from cocktails of fruits and vegetables with the abundance of ubiquinone contained in the supplement-HEPATOSAAB. 